
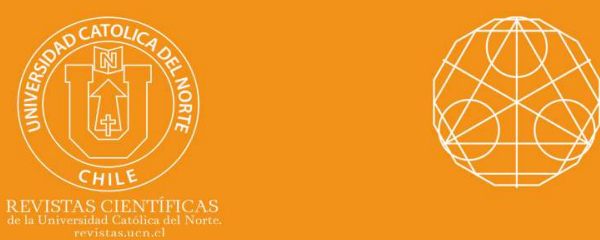

\title{
SD-prime cordial labeling of alternate k-polygonal snake of various types
}

U. M. Prajapati ${ }^{1^{*}}$

Anit Vantiya ${ }^{2}$ (1) orcid.org/0000-0002-0276-5491

${ }^{1}$ St. Xavier's College (Autonomous), Dept. of Mathematics, Ahmedabad, GJ, India.

udayan64@yahoo.com

${ }^{2}$ Gujarat University, Dept. of Mathematics, Ahmedabad, GJ, India

vavantiya@yahoo.co.in

Received: 23 February 2020 | Accepted: 13 December 2020

\section{Abstract:}

Let $f: V(G) \rightarrow\{1,2, \ldots,|V(G)|\}$ be a bijection, and let us denote $S=f(u)+f(v)$ and $D=$ $|f(u)-f(v)|$ for every edge uv in $E(G)$. Let $f^{\prime}$ be the induced edge labeling, induced by the vertex labeling $f$, defined as $f^{\prime}: E(G) \rightarrow\{0,1\}$ such that for any edge uv in $E(G), f^{\prime}(u v)=1$ if $\operatorname{gcd}(S, D)=1$, and $f^{\prime}(\mathrm{Uv})=0$ otherwise. Let ef $(0)$ and ef $(1)$ be the number of edges labeled with 0 and 1 respectively. $f$ is $S D$-prime cordial labeling if $\mid e f^{\prime}(0)-e f^{\prime}(1) / \leq 1$ and $G$ is $S D$-prime cordial graph if it admits SD-prime cordial labeling. In this paper, we have discussed the SD-prime cordial labeling of alternate $k$-polygonal snake graphs of type-1, type- 2 and type-3.

Keywords: SD-prime cordial graph; Triangular snake; Alternate quadrilateral snake; n-polygonal snake; Alternate k-polygonal snake.

MSC (2020): $05 C 78$.

Cite this article as (IEEE citation style):

U. M. Prajapati and A. Vantiya, "SD-prime cordial labeling of alternate k-polygonal snake of various types", Proyecciones (Antofagasta, On line), vol. 40, no. 3, pp. 619-634, 2021, doi: 10.22199/issn.0717-6279-4015

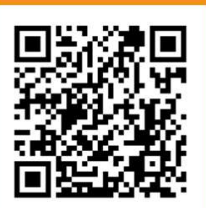

Article copyright: (C) 2021 U. M. Prajapati and Anit Vantiya. This is an open access article distributed under the terms of the Creative Commons License, which permits unrestricted use and distribution provided the original author and source are credited.

*Corresponding author 


\section{Introduction}

Let $G=(V(G), E(G))$ be a simple, finite and undirected graph of order $|V(G)|$ and size $|E(G)|$. For standard terminology of Graph Theory, we used [1]. For all detailed survey of graph labeling we refer [2]. Lau, Chu, Suhadak, Foo, and $\mathrm{Ng}[3]$ have introduced SD-prime cordial labeling and they proved behaviour of several graphs like path, complete bipartite graph, star, double star, wheel, fan, double fan and ladder. Lourdusamy and Patrick [4] proved that $S^{\prime}\left(K_{1, n}\right), D_{2}\left(K_{1, n}\right), S\left(K_{1, n}\right), D S\left(K_{1, n}\right), S^{\prime}\left(B_{n, n}\right)$, $D_{2}\left(B_{n, n}\right), T L_{n}, D S\left(B_{n, n}\right), S\left(B_{n, n}\right), K_{1,3} \star K_{1, n}, C H_{n}, F l_{n}, P_{n}^{2}, T\left(P_{n}\right), T\left(C_{n}\right)$, $Q_{n}, A\left(T_{n}\right), P_{n} \odot K_{1}, C_{n} \odot K_{1}, J_{n}$ and the graph obtained by duplication of each vertex and cycle by an edge are SD-prime cordial. Lourdusamy, Wency and Patrick [5] proved that the union of star and path graphs, subdivision of comb graph, subdivision of ladder graph and the graph obtained by attaching star graph at one end of the path are SD-prime cordial graphs. They proved that the union of two SD-prime cordial graphs need not be SD-prime cordial graph. Also, they proved that given a positive integer $n$, there is SD-prime cordial graph $G$ with $n$ vertices. Prajapati and Vantiya [8] proved that $T_{n}(n \neq 3), A\left(T_{n}\right), Q_{n}, A\left(Q_{n}\right), D T_{n}, D A\left(T_{n}\right), D Q_{n}$ and $D A\left(Q_{n}\right)$ are SD-prime cordial. Prajapati and Vantiya [9] proved that $S\left(T_{n}\right), S\left(A\left(T_{n}\right)\right)$, $S\left(Q_{n}\right)$ and $S\left(A\left(Q_{n}\right)\right)$ are SD-prime cordial. In this paper, we investigate the SD-prime cordial labeling behavior of alternate k-polygonal snake graphs.

Definition 1.1 (3). For a graph $G$, a bijection $f: V(G) \rightarrow\{1,2, \ldots,|V(G)|\}$ induces an edge labeling $f^{\prime}: E(G) \rightarrow\{0,1\}$ such that for any edge uv in $G, f^{\prime}(u v)=1$ if $\operatorname{gcd}(S, D)=1$, and $f^{\prime}(u v)=0$ otherwise, where $S=f(u)+f(v)$ and $D=|f(u)-f(v)|$ for every edge $u v$ in $E(G)$. Let $e_{f^{\prime}}(0)$ and $e_{f^{\prime}}(1)$ be the number of edges labeled with 0 and 1 respectively. The labeling $f$ is called $S D$-prime cordial labeling if $\left|e_{f^{\prime}}(0)-e_{f^{\prime}}(1)\right| \leq 1$. $G$ is called $S D$-prime cordial graph if it admits SD-prime cordial labeling.

Definition $1.2(2)$. A triangular snake $T_{n}$ is obtained from the path $P_{n}$ by replacing every edge of a path by a triangle $C_{3}$. That is, it is obtained from a path $u_{1}, u_{2}, \ldots, u_{n}$ by joining $u_{i}$ and $u_{i+1}$ to a new vertex $w_{i}$ for $i=1,2, \ldots, n-1$.

Definition 1.3 (2). A quadrilateral snake $Q_{n}$ is obtained from the path $P_{n}: u_{1}, u_{2}, \ldots, u_{n}$ by replacing every edge of a path by a cycle $C_{4}$, such that each pair $u_{i}, u_{i+1}$ remains adjacent for all $i=1,2, \ldots, n$. That is, 
it is obtained from a path $u_{1}, u_{2}, \ldots, u_{n}$ by joining $u_{i}$ and $u_{i+1}$ to new vertex $v_{i}$ and $w_{i}$ respectively, and then joining $v_{i}$ and $w_{i}$ by an edge, for $i=1,2, \ldots, n-1$.

Definition 1.4. [6] A k-polygonal snake is obtained by replacing every edge of a path $P_{n}: u_{1}, u_{2}, \ldots, u_{n}$ by $k$-cycle $C_{k}$ for $k \geq 3$, such that each pair $u_{i}, u_{i+1}$ remains adjacent for all $i=1,2, \ldots, n$. It is denoted by $S_{n}\left(C_{k}\right)$.

Note that, $S_{n}\left(C_{3}\right)$ is the triangular snake graph and $S_{n}\left(C_{4}\right)$ is the quadrilateral snake graph.

Definition 1.5. [7] An alternate k-polygonal snake is obtained by replacing every alternate edge of a path $P_{n}: u_{1}, u_{2}, \ldots, u_{n}$ by $k$-cycle $C_{k}$ for $k \geq 3$, such that each pair $u_{i}, u_{i+1}$ remains adjacent for all $i=1,2, \ldots, n$. It is denoted by $A S_{n}\left(C_{k}\right)$.

The alternate k-polygonal snake graph $A S_{n}\left(C_{k}\right)$ is obtained from path $P_{n}$ by joining $u_{i}$ and $u_{i+1}$, alternatively, (that is for all odd i's or for all even i's) by the path $v_{i, 1}, v_{i, 2}, \ldots, v_{i, k-2}$, where $k \geq 3$. There can be three non-isomorphic alternate k-polygonal snakes, we define them as follow.

Definition 1.6. An alternate k-polygonal snake of type-1 is an alternate $k$-polygonal snake in which $n$ is even and the edge $u_{i} u_{i+1}$ is replaced by $k$-cycle, for every odd $i$. It is denoted by $A S_{n}^{1}\left(C_{k}\right)$.

Let $V\left(A S_{n}^{1}\left(C_{k}\right)\right)=V\left(P_{n}\right) \cup\left\{v_{i, j}: i\right.$ is odd and $1 \leq i \leq n-1,1 \leq j \leq$ $k-2\}$ and $E\left(A S_{n}^{1}\left(C_{k}\right)\right)=E\left(P_{n}\right) \cup\left\{u_{i} v_{i, 1}, v_{i, k-2} u_{i+1}: i\right.$ is odd and $1 \leq i \leq$ $n-1\} \cup\left\{v_{i, j} v_{i, j+1}: i\right.$ is odd and $\left.1 \leq i \leq n-1,1 \leq j \leq k-3\right\} . A S_{n}^{1}\left(C_{k}\right)$ is of order $\frac{n k}{2}$ and size $\frac{n(k+1)-2}{2}$. For an example, see the figure 1 . 


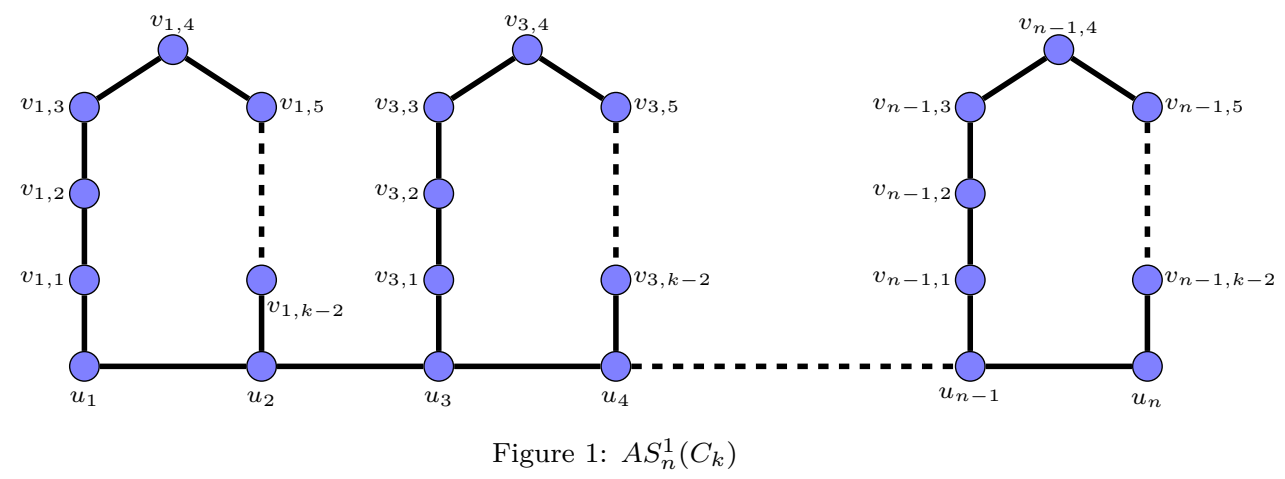

Definition 1.7. An alternate k-polygonal snake of type-2 is an alternate $k$-polygonal snake in which $n$ is odd and the edge $u_{i} u_{i+1}$ is replaced by $k$-cycle, for every odd $i$. It is denoted by $A S_{n}^{2}\left(C_{k}\right)$.

Define $V\left(A S_{n}^{2}\left(C_{k}\right)\right)$ and $E\left(A S_{n}^{2}\left(C_{k}\right)\right)$ as per the type-1 above. $A S_{n}^{2}\left(C_{k}\right)$ is of order $\frac{(n-1) k+2}{2}$ and size $\frac{(n-1)(k+1)}{2}$. For an example, see the figure 2 .
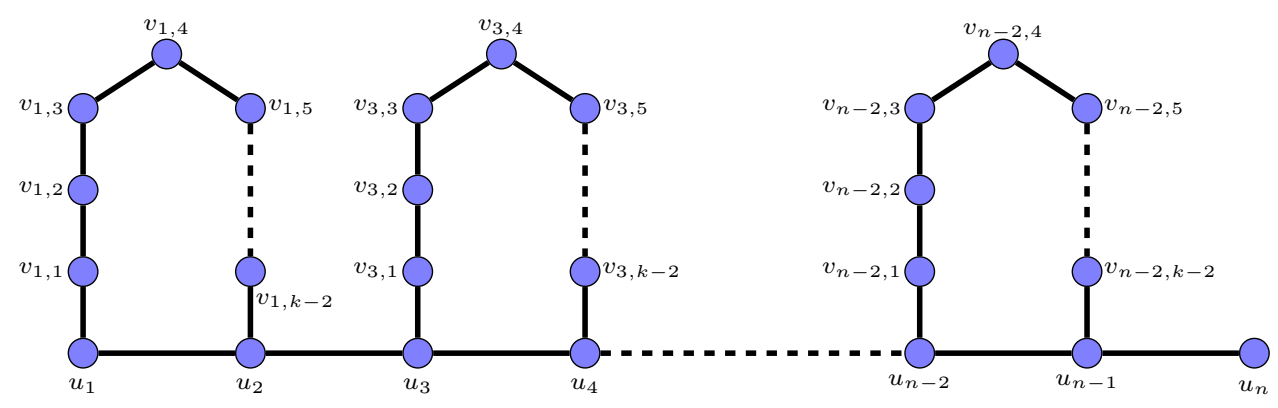

Figure 2: $A S_{n}^{2}\left(C_{k}\right)$

Definition 1.8. An alternate k-polygonal snake of type-3 is an alternate $k$-polygonal snake in which $n$ is even and the edge $u_{i} u_{i+1}$ is replaced by $k$-cycle, for every even $i$. It is denoted by $A S_{n}^{3}\left(C_{k}\right)$.

Let $V\left(A S_{n}^{3}\left(C_{k}\right)\right)=V\left(P_{n}\right) \cup\left\{v_{i, j}: i\right.$ is even and $1 \leq i \leq n-1,1 \leq j \leq$ $k-2\}$ and $E\left(A S_{n}^{3}\left(C_{k}\right)\right)=E\left(P_{n}\right) \cup\left\{u_{i} v_{i, 1}, v_{i, k-2} u_{i+1}: i\right.$ is even and $1 \leq$ $i \leq n-1\} \cup\left\{v_{i, j} v_{i, j+1}: i\right.$ is even and $\left.1 \leq i \leq n-1,1 \leq j \leq k-3\right\}$. $A S_{n}^{3}\left(C_{k}\right)$ is of order $\frac{(n-2) k+4}{2}$ and size $\frac{(n-2) k+n}{2}$. For an example, see the figure 3 . 


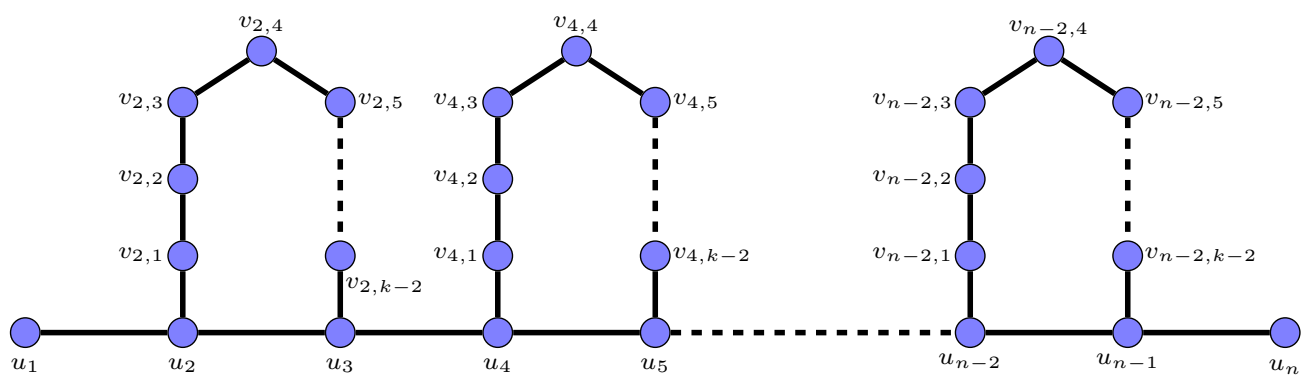

Figure 3: $A S_{n}^{3}\left(C_{k}\right)$

\section{Main Results}

Prajapati and Vantiya [8] proved that alternate triangular snake $A\left(T_{n}\right)$ and alternate quadrilateral snake $A\left(Q_{n}\right)$ are SD-prime cordial. Thus for $k=3$ and $4 ; A S_{n}^{1}\left(C_{k}\right), A S_{n}^{2}\left(C_{k}\right)$ and $A S_{n}^{3}\left(C_{k}\right)$ are SD-prime cordial. Here we consider the cases for remaining arbitrary values of $k \in N$.

Theorem 2.1. The graph $A S_{n}^{1}\left(C_{k}\right)$ is $S D$-prime cordial, for $k \equiv 1(\bmod 4), k \geq 5$.

Proof 2.1. Define $f: V\left(A S_{n}^{1}\left(C_{k}\right)\right) \rightarrow\left\{1,2, \ldots, \frac{n k}{2}\right\}$ as follows:

$$
\begin{array}{lll}
f\left(u_{i}\right)=\frac{k i}{2}-\frac{k-2}{4}+\frac{k-2}{4}(-1)^{i}, & \text { if } 1 \leq i \leq n ; \\
f\left(v_{i, 1}\right)=\frac{k i}{2}-\frac{k}{2}+3, & \text { if } i \text { is odd, } 1 \leq i \leq n-1 ; \\
f\left(v_{i, 2}\right)=\frac{k i}{2}-\frac{k}{2}+2, & \text { if } i \text { is odd, } 1 \leq i \leq n-1 ; \\
f\left(v_{i, 3}\right)=\frac{k i}{2}-\frac{k}{2}+4, & \text { if } i \text { is odd, } 1 \leq i \leq n-1 ; \\
f\left(v_{i, j}\right)=\frac{k i}{2}-\frac{k}{2}+j+1, & \text { if } j \equiv 0(\bmod 4) \text { or } \\
& j \equiv 3(\bmod 4), 4 \leq j \leq k-2, \\
& \text { and } i \text { is odd } 1 \leq i \leq n-1 ; \\
f\left(v_{i, j}\right)=\frac{k i}{2}-\frac{k}{2}+j+2, & \text { if } j \equiv 1(\bmod 4), 5 \leq j \leq k-2, \\
& \text { and } i \text { is odd } 1 \leq i \leq n-1 ; \\
f\left(v_{i, j}\right)=\frac{k i}{2}-\frac{k}{2}+j, & \text { if } j \equiv 2(\bmod 4), 6 \leq j \leq k-2, \\
& \text { and } i \text { is odd } 1 \leq i \leq n-1 .
\end{array}
$$


Then $e_{f^{\prime}}(0)=\left\lceil\frac{n(k+1)-2}{4}\right\rceil$ and $e_{f^{\prime}}(1)=\left\lfloor\frac{n(k+1)-2}{4}\right\rfloor$.

Thus $\left|e_{f^{\prime}}(0)-e_{f^{\prime}}(1)\right| \leq 1$.

Hence $A S_{n}^{1}\left(C_{k}\right)$ is $S D$-prime cordial, for $k \equiv 1(\bmod 4), k \geq 5$.

Theorem 2.2. The graph $A S_{n}^{2}\left(C_{k}\right)$ is $S D$-prime cordial, for $k \equiv 1(\bmod 4), k \geq 5$.

Proof 2.2. Define $f: V\left(A S_{n}^{2}\left(C_{k}\right)\right) \rightarrow\left\{1,2, \ldots, \frac{(n-1) k+2}{2}\right\}$ as per the above theorem.

Then $e_{f^{\prime}}(0)=e_{f^{\prime}}(1)=\frac{(n-1)(k+1)}{4}$.

Thus $\left|e_{f^{\prime}}(0)-e_{f^{\prime}}(1)\right| \leq 1$.

Hence $A S_{n}^{2}\left(C_{k}\right)$ is $S D$-prime cordial, for $k \equiv 1(\bmod 4), k \geq 5$.

Theorem 2.3. The graph $A S_{n}^{3}\left(C_{k}\right)$ is $S D$-prime cordial, for $k \equiv 1(\bmod 4), k \geq 5$.

Proof 2.3. Define $f: V\left(A S_{n}^{3}\left(C_{k}\right)\right) \rightarrow\left\{1,2, \ldots, \frac{(n-1) k+4}{2}\right\}$ as follows:

$$
\begin{array}{rlrl}
f\left(u_{i}\right)=\frac{k i}{2}-\frac{3 k}{4}-\frac{k}{4}(-1)^{i} & \\
+\frac{3}{2}+\frac{1}{2}(-1)^{i}, & & \text { if } 1 \leq i \leq n ; \\
f\left(v_{i, j}\right)=\frac{k i}{2}-k+j+2, & \text { if } j \equiv 0(\bmod 4) \text { or } \\
& j \equiv 3(\bmod 4), 3 \leq j \leq k-2, \\
& \text { and } i \text { is even, } 1 \leq i \leq n-1 ; \\
f\left(v_{i, j}\right)=\frac{k i}{2}-k+j+3, & \text { if } j \equiv 1(\bmod 4), 1 \leq j \leq k-2, \\
& \text { and } i \text { is even, } 1 \leq i \leq n-1 ; \\
f\left(v_{i, j}\right)=\frac{k i}{2}-k+j+1, & \text { if } j \equiv 2(\bmod 4), 2 \leq j \leq k-2, \\
& \text { and } i \text { is even, } 1 \leq i \leq n-1 .
\end{array}
$$

Then $e_{f^{\prime}}(0)=\left\lfloor\frac{(n-2) k+n}{4}\right\rfloor$ and $e_{f^{\prime}}(1)=\left\lceil\frac{(n-2) k+n}{4}\right\rceil$.

Thus $\left|e_{f^{\prime}}(0)-e_{f^{\prime}}(1)\right| \leq 1$.

Hence $A S_{n}^{3}\left(C_{k}\right)$ is $S D$-prime cordial, for $k \equiv 1(\bmod 4), k \geq 5$.

Theorem 2.4. The graph $A S_{n}^{1}\left(C_{6}\right)$ is $S D$-prime cordial. 
Proof 2.4. Define $f: V\left(A S_{n}^{1}\left(C_{6}\right)\right) \rightarrow\{1,2, \ldots, 3 n\}$ as follows:

$$
\begin{aligned}
& f\left(u_{i}\right)= \begin{cases}3 i-2, & \text { if } i \text { is odd, } 1 \leq i \leq n ; \\
3 i-1, & \text { if } i \text { is even, } 1 \leq i \leq n\end{cases} \\
& f\left(v_{i, 1}\right)= \begin{cases}3 i+1, & \text { if } i \equiv 1(\bmod 4), 1 \leq i \leq n-1 ; \\
3 i-1, & \text { if } i \equiv 3(\bmod 4), 1 \leq i \leq n-1\end{cases} \\
& f\left(v_{i, 2}\right)= \begin{cases}3 i+3, & \text { if } i \equiv 1(\bmod 4), 1 \leq i \leq n-1 ; \\
3 i, & \text { if } i \equiv 3(\bmod 4), 1 \leq i \leq n-1 ;\end{cases} \\
& f\left(v_{i, 3}\right)= \begin{cases}3 i, & \text { if } i \equiv 1(\bmod 4), 1 \leq i \leq n-1 ; \\
3 i+1, & \text { if } i \equiv 3(\bmod 4), 1 \leq i \leq n-1 ;\end{cases} \\
& f\left(v_{i, 4}\right)= \begin{cases}3 i-1, & \text { if } i \equiv 1(\bmod 4), 1 \leq i \leq n-1 ; \\
3 i+3, & \text { if } i \equiv 3(\bmod 4), 1 \leq i \leq n-1 .\end{cases}
\end{aligned}
$$

Then $e_{f^{\prime}}(0)=\left\lfloor\frac{7 n-2}{4}\right\rfloor$ and $e_{f^{\prime}}(1)=\left\lceil\frac{7 n-2}{4}\right\rceil$.

Thus, $\left|e_{f^{\prime}}(0)-e_{f^{\prime}}(1)\right| \leq 1$. Hence $A S_{n}^{1}\left(C_{6}\right)$ is SD-prime cordial.

Theorem 2.5. The graph $A S_{n}^{2}\left(C_{6}\right)$ is $S D$-prime cordial.

Proof 2.5. Define $f: V\left(A S_{n}^{2}\left(C_{6}\right)\right) \rightarrow\left\{1,2, \ldots, \frac{6 n-4}{2}\right\}$ as per the previous theorem.

Then $e_{f^{\prime}}(0)=\left\lceil\frac{7(n-1)}{4}\right\rceil$ and $e_{f^{\prime}}(1)=\left\lfloor\frac{7(n-1)}{4}\right\rfloor$.

Thus, $\left|e_{f^{\prime}}(0)-e_{f^{\prime}}(1)\right| \leq 1$. Hence $A S_{n}^{2}\left(C_{6}\right)$ is $S D$-prime cordial.

Theorem 2.6. The graph $A S_{n}^{3}\left(C_{6}\right)$ is $S D$-prime cordial.

Proof 2.6. Define $f: V\left(A S_{n}^{3}\left(C_{6}\right)\right) \rightarrow\left\{1,2, \ldots, \frac{6 n-8}{2}\right\}$ as follows:

$$
\begin{aligned}
& f\left(u_{i}\right)= \begin{cases}1, & \text { if } i=1 ; \\
3 i-3, & \text { if } i \text { is odd }, 3 \leq i \leq n ; \\
3 i-4, & \text { if } i \text { is even, } 1 \leq i \leq n ;\end{cases} \\
& f\left(v_{i, 1}\right)= \begin{cases}5, & \text { if } i=2 ; \\
3 i-3, & \text { if } i \equiv 2(\bmod 4), 6 \leq i \leq n-1 ; \\
3 i-1, & \text { if } i \equiv 0(\bmod 4), 1 \leq i \leq n-1 ;\end{cases} \\
& f\left(v_{i, 2}\right)= \begin{cases}7, & \text { if } i=2 ; \\
3 i-2, & \text { if } i \equiv 2(\bmod 4), 6 \leq i \leq n-1 ; \\
3 i+1, & \text { if } i \equiv 0(\bmod 4), 1 \leq i \leq n-1 ;\end{cases}
\end{aligned}
$$


$f\left(v_{i, 3}\right)= \begin{cases}4, & \text { if } i=2 ; \\ 3 i-1, & \text { if } i \equiv 2(\bmod 4), 6 \leq i \leq n-1 ; \\ 3 i-2, & \text { if } i \equiv 0(\bmod 4), 1 \leq i \leq n-1 ;\end{cases}$

$f\left(v_{i, 4}\right)= \begin{cases}3, & \text { if } i=2 ; \\ 3 i+1, & \text { if } i \equiv 2(\bmod 4), 6 \leq i \leq n-1 ; \\ 3 i-3, & \text { if } i \equiv 0(\bmod 4), 1 \leq i \leq n-1 .\end{cases}$

Then $e_{f^{\prime}}(0)=\left\lceil\frac{7 n-12}{4}\right\rceil$ and $e_{f^{\prime}}(1)=\left\lfloor\frac{7 n-12}{4}\right\rfloor$.

Thus, $\left|e_{f^{\prime}}(0)-e_{f^{\prime}}(1)\right| \leq 1$. Hence $A S_{n}^{3}\left(C_{6}\right)$ is $S D$-prime cordial.

Theorem 2.7. The graph $A S_{n}^{1}\left(C_{k}\right)$ is $S D$-prime cordial, for $k \equiv 2(\bmod 4), k \geq 10$.

Proof 2.7. Define $f: V\left(A S_{n}^{1}\left(C_{k}\right)\right) \rightarrow\left\{1,2, \ldots, \frac{n k}{2}\right\}$ as follows:

$f\left(u_{i}\right)= \begin{cases}\frac{k i}{2}-\frac{k}{2}+1, & \text { if } i \text { is odd, } 1 \leq i \leq n ; \\ \frac{k i}{2}-1, & \text { if } i \text { is even, } 1 \leq i \leq n ;\end{cases}$

$f\left(v_{i, 1}\right)= \begin{cases}\frac{k i}{2}-\frac{k}{2}+4, & \text { if } i \equiv 1(\bmod 4), 1 \leq i \leq n-1 ; \\ \frac{k i}{2}-\frac{k}{2}+2, & \text { if } i \equiv 3(\bmod 4), 1 \leq i \leq n-1 ;\end{cases}$

$f\left(v_{i, 2}\right)=\left\{\begin{array}{l}\frac{k i}{2}-\frac{k}{2}+2, \quad \text { if } i \equiv 1(\bmod 4), 1 \leq i \leq n-1 ; \\ \frac{k i}{2}-\frac{k}{2}+3,\end{array}\right.$

$\begin{cases}\frac{k i}{2}-\frac{k}{2}+3, & \text { if } i \equiv 3(\bmod 4), 1 \leq i \leq n-1 ;\end{cases}$

$f\left(v_{i, 3}\right)= \begin{cases}\frac{k i}{2}-\frac{k}{2}+5, & \text { if } i \equiv 1(\bmod 4), 1 \leq i \leq n-1 ; \\ \frac{k i}{2}-\frac{k}{2}+4, & \text { if } i \equiv 3(\bmod 4), 1 \leq i \leq n-1 ;\end{cases}$

$f\left(v_{i, 4}\right)=\left\{\begin{array}{l}\frac{k i}{2}-\frac{k}{2}+3, \quad \text { if } i \equiv 1(\bmod 4), 1 \leq i \leq n-1 ; \\ \text { kif }\end{array}\right.$

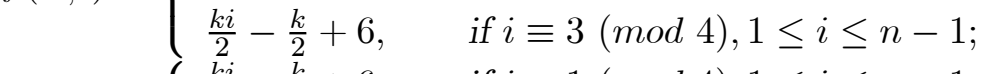

$f\left(v_{i, 5}\right)= \begin{cases}\frac{k i}{2}-\frac{k}{2}+6, & \text { if } i \equiv 1(\bmod 4), 1 \leq i \leq n-1 ; \\ \frac{k i}{2}-\frac{k}{2}+5, & \text { if } i \equiv 3(\bmod 4), 1 \leq i \leq n-1 ;\end{cases}$

$f\left(v_{i, j}\right)=\frac{k i}{2}-\frac{k}{2}+j+1, \quad$ if $j \equiv 2(\bmod 4)$ or $j \equiv 3(\bmod 4)$,

$f\left(v_{i, j}\right)=\frac{k i}{2}-\frac{k}{2}+j+2, \quad$ if $j \equiv 0(\bmod 4), 8 \leq j \leq k-2$, and $i$ is odd, $1 \leq i \leq n-1$;

$f\left(v_{i, j}\right)=\frac{k i}{2}-\frac{k}{2}+j, \quad$ if $j \equiv 1(\bmod 4), 9 \leq j \leq k-2$, and $i$ is odd, $1 \leq i \leq n-1$. 
Then $e_{f^{\prime}}(0)=\left\lfloor\frac{n(k+1)-2}{4}\right\rfloor$ and $e_{f^{\prime}}(1)=\left\lceil\frac{n(k+1)-2}{4}\right\rceil$.

Thus, $\left|e_{f^{\prime}}(0)-e_{f^{\prime}}(1)\right| \leq 1$.

Hence $A S_{n}^{1}\left(C_{k}\right)$ is $S D$-prime cordial, for $k \equiv 2(\bmod 4), k \geq 10$.

\section{Illustration-1:}

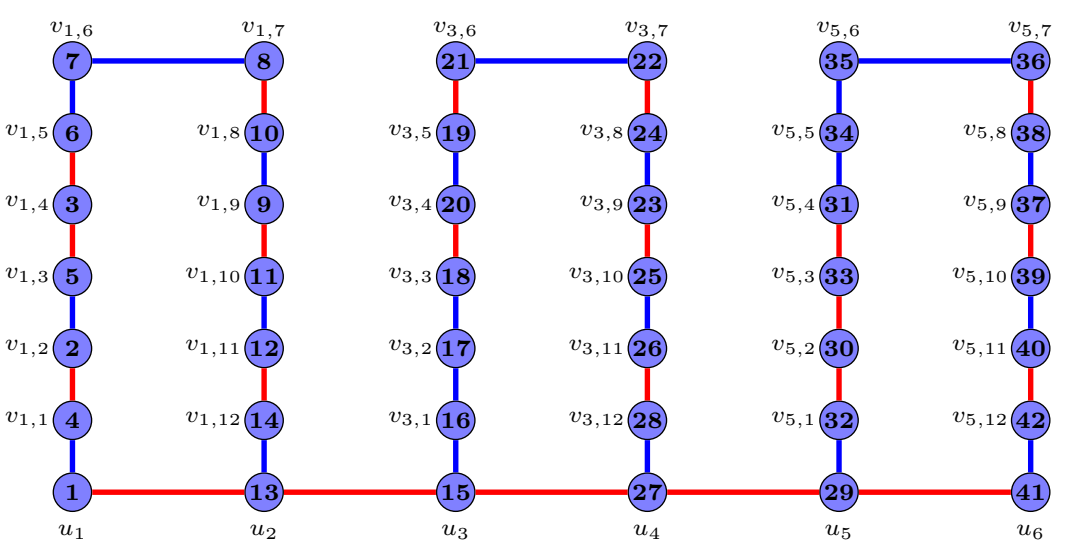

Figure 4: $A S_{6}^{1}\left(C_{14}\right), n=6, k=14, e_{f^{\prime}}(1)=e_{f^{\prime}}(0)=22$

Theorem 2.8. The graph $A S_{n}^{2}\left(C_{k}\right)$ is $S D$-prime cordial, for $k \equiv 2(\bmod 4), k \geq 10$.

Proof 2.8. Define $f: V\left(A S_{n}^{2}\left(C_{k}\right)\right) \rightarrow\left\{1,2, \ldots, \frac{(n-1) k+2}{2}\right\}$ as per the previous theorem. Then $e_{f^{\prime}}(0)=\left\lceil\frac{(n-1)(k+1)}{4}\right\rceil$ and $e_{f^{\prime}}(1)=\left\lfloor\frac{(n-1)(k+1)}{4}\right\rfloor$.

Thus, $\left|e_{f^{\prime}}(0)-e_{f^{\prime}}(1)\right| \leq 1$.

Hence $A S_{n}^{2}\left(C_{k}\right)$ is $S D$-prime cordial, for $k \equiv 2(\bmod 4), k \geq 10$.

Theorem 2.9. The graph $A S_{n}^{3}\left(C_{k}\right)$ is $S D$-prime cordial, for $k \equiv 2(\bmod 4), k \geq 10$.

Proof 2.9. Define $f: V\left(A S_{n}^{3}\left(C_{k}\right)\right) \rightarrow\left\{1,2, \ldots, \frac{(n-1) k+4}{2}\right\}$ as follows: 


$$
\begin{aligned}
& \begin{cases}1, & \text { if } i=1 ; \\
\frac{k i}{2}-\frac{k}{2}, & \text { if } i \text { is }\end{cases} \\
& f\left(u_{i}\right)= \begin{cases}\frac{k i}{2}-\frac{k}{2}, & \text { if } i \text { is odd, } 3 \leq i \leq n ; \\
\frac{\pi}{2}-k+2, & \text { if } i \text { is even, } 1 \leq i \leq n ;\end{cases} \\
& f\left(v_{i, 1}\right)= \begin{cases}\frac{k i}{2}-k+2, & \text { if } i \text { is even, } 1 \leq i \leq n ; \\
\frac{k i}{2}-k+5, & \text { if } i \equiv 0(\bmod 4), 1 \leq i \leq n-1 \text { or } i=2 ;\end{cases} \\
& f\left(v_{i, 2}\right)= \begin{cases}\frac{k i}{2}-k+3, & \text { if } i \equiv 2(\bmod 4), 6 \leq i \leq n-1 ; \\
\frac{k i}{2}-k+3, & \text { if } i \equiv 0(\bmod 4), 1 \leq i \leq n-1 \text { or } i=2 ; \\
\frac{k i}{2}-k+4, & \text { if } i \equiv 2(\bmod 4), 6 \leq i \leq n\end{cases} \\
& \left\{\begin{array}{l}
\frac{k i}{2}-k+4, \quad \text { if } i \equiv 2(\bmod 4), 6 \leq i \leq n-1 ;
\end{array}\right. \\
& f\left(v_{i, 3}\right)= \begin{cases}\frac{k i}{2}-k+6, & \text { if } i \equiv 0(\bmod 4), 1 \leq i \leq n-1 \text { or } i=2 ; \\
\frac{k i}{2}-k+5, & \text { if } i \equiv 2(\bmod 4), 6 \leq i \leq n-1 ; \\
\frac{k i}{2}-k+4, & \text { if } i \equiv 0(\bmod 4), 1 \leq i \leq n-1 \text { or } i=2 ;\end{cases} \\
& f\left(v_{i, 4}\right)=\left\{\begin{array}{l}
\frac{k i}{2}-k+7, \quad \text { if } i \equiv 2(\bmod 4), 6 \leq i \leq n-1 ;
\end{array}\right. \\
& f\left(v_{i, 5}\right)= \begin{cases}\frac{k i}{2}-k+7, & \text { if } i \equiv 0(\bmod 4), 1 \leq i \leq n-1 \\
\frac{k i}{2}-k+6, & \text { if } i \equiv 2(\bmod 4), 6 \leq i \leq n-1\end{cases} \\
& f\left(v_{i, j}\right)=\frac{k i}{2}-k+j+2, \quad \text { if } j \equiv 2(\bmod 4) \text { or } j \equiv 3(\bmod 4) \text {, } \\
& f\left(v_{i, j}\right)=\frac{k i}{2}-k+j+3, \quad \text { if } j \equiv 0(\bmod 4), 8 \leq j \leq k-2 \text {, } \\
& \text { and } i \text { is even, } 1 \leq i \leq n-1 \text {; } \\
& f\left(v_{i, j}\right)=\frac{k i}{2}-k+j+1, \quad \text { if } j \equiv 1(\bmod 4), 9 \leq j \leq k-2, \\
& \text { and } i \text { is even, } 1 \leq i \leq n-1 \text {. }
\end{aligned}
$$

Then $e_{f^{\prime}}(0)=\left\lceil\frac{(n-2) k+n}{4}\right\rceil$ and $e_{f^{\prime}}(1)=\left\lfloor\frac{(n-2) k+n}{4}\right\rfloor$, for $n \geq 4$.

Thus, $\left|e_{f^{\prime}}(0)-e_{f^{\prime}}(1)\right| \leq 1$.

Hence $A S_{n}^{3}\left(C_{k}\right)$ is $S D$-prime cordial, for $k \equiv 2(\bmod 4), k \geq 10$.

Theorem 2.10. The graph $A S_{n}^{1}\left(C_{k}\right)$ is $S D$-prime cordial, for $k \equiv 3(\bmod 4), k \geq 7$.

Proof 2.10. Define $f: V\left(A S_{n}^{1}\left(C_{k}\right)\right) \rightarrow\left\{1,2, \ldots, \frac{n k}{2}\right\}$ as follows: 


$$
\begin{array}{lll}
f\left(u_{i}\right)=\frac{k i}{2}-\frac{k-2}{4}+\frac{k-2}{4}(-1)^{i}, & & \text { if } 1 \leq i \leq n ; \\
f\left(v_{i, 1}\right)=\frac{k i}{2}-\frac{k}{2}+4, & & \text { if } i \text { is odd, } 1 \leq i \leq n-1 ; \\
f\left(v_{i, 2}\right)=\frac{k i}{2}-\frac{k}{2}+2, & & \text { if } i \text { is odd } 1 \leq i \leq n-1 ; \\
f\left(v_{i, 4}\right)=\frac{k i}{2}-\frac{k}{2}+3, & \text { if } i \text { is odd, } 1 \leq i \leq n-1 ; \\
f\left(v_{i, j}\right)=\frac{k i}{2}-\frac{k}{2}+j+1, & \text { if } j \equiv 1(\bmod 4) \text { or } \\
& & j \equiv 2(\bmod 4), 5 \leq j \leq k-2, \\
& \text { and } i \text { is odd } 1 \leq i \leq n-1 ; \\
f\left(v_{i, j}\right)=\frac{k i}{2}-\frac{k}{2}+j+2, & \text { if } j \equiv 3(\bmod 4), 3 \leq j \leq k-2, \\
& \text { and } i \text { is odd, } 1 \leq i \leq n-1 ; \\
f\left(v_{i, j}\right)=\frac{k i}{2}-\frac{k}{2}+j, & \text { if } j \equiv 0(\bmod 4), 8 \leq j \leq k-2, \\
& \text { and } i \text { is odd } 1 \leq i \leq n-1 .
\end{array}
$$

Then $e_{f^{\prime}}(0)=\left\lceil\frac{n(k+1)-2}{4}\right\rceil$ and $e_{f^{\prime}}(1)=\left\lfloor\frac{n(k+1)-2}{4}\right\rfloor$.

Thus, $\left|e_{f^{\prime}}(0)-e_{f^{\prime}}(1)\right| \leq 1$.

Hence $A S_{n}^{1}\left(C_{k}\right)$ is $S D$-prime cordial, for $k \equiv 3(\bmod 4), k \geq 7$.

Theorem 2.11. The graph $A S_{n}^{2}\left(C_{k}\right)$ is SD-prime cordial, for $k \equiv 3(\bmod 4), k \geq 7$.

Proof 2.11. Define $f: V\left(A S_{n}^{2}\left(C_{k}\right)\right) \rightarrow\left\{1,2, \ldots, \frac{(n-1) k+2}{2}\right\}$ as per the previous theorem. Then $e_{f^{\prime}}(0)=e_{f^{\prime}}(1)=\frac{(n-1)(k+1)}{4}$.

Thus, $\left|e_{f^{\prime}}(0)-e_{f^{\prime}}(1)\right| \leq 1$.

Hence $A S_{n}^{2}\left(C_{k}\right)$ is $S D$-prime cordial, for $k \equiv 3(\bmod 4), k \geq 7$. 


\section{Illustration-2:}

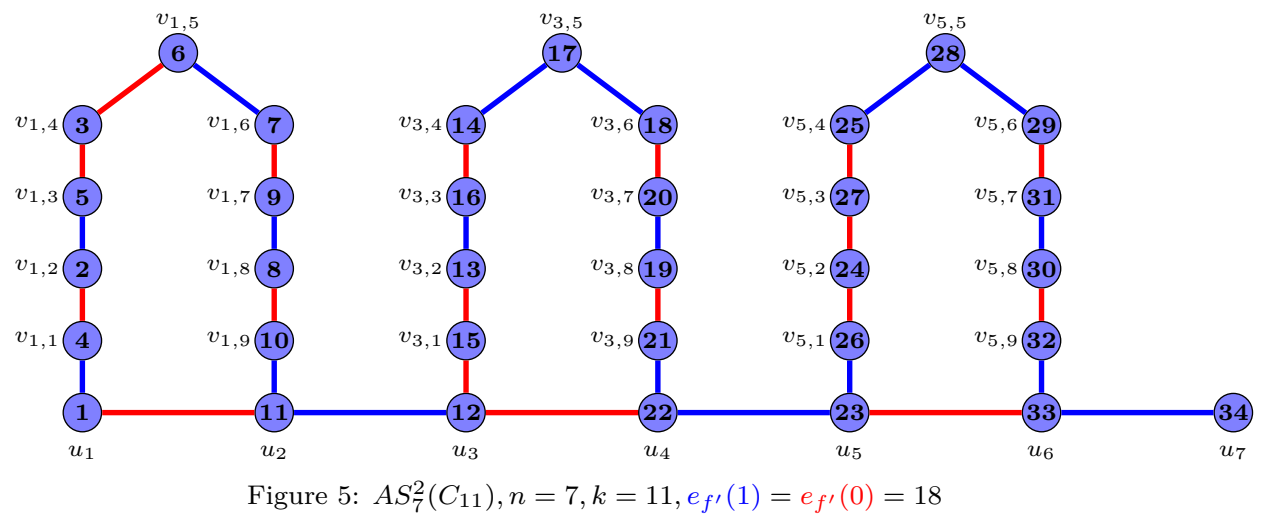

Theorem 2.12. The graph $A S_{n}^{3}\left(C_{k}\right)$ is $S D$-prime cordial, for $k \equiv 3(\bmod 4), k \geq 7$.

Proof 2.12. Define $f: V\left(A S_{n}^{3}\left(C_{k}\right)\right) \rightarrow\left\{1,2, \ldots, \frac{(n-1) k+4}{2}\right\}$ as follows:

$$
\begin{aligned}
& f\left(u_{i}\right)= \begin{cases}\frac{k i}{2}-\frac{k}{2}+1, & \text { if } i \text { is odd, } 1 \leq i \leq n ; \\
\frac{k i}{2}-k+2, & \text { if } i \text { is even, } 1 \leq i \leq n ;\end{cases} \\
& f\left(v_{i, 1}\right)=\frac{k i}{2}-k+5, \quad \text { if } i \text { is even, } 1 \leq i \leq n-1 \text {; } \\
& f\left(v_{i, 2}\right)=\frac{k i}{2}-k+3, \quad \text { if } i \text { is even, } 1 \leq i \leq n-1 \text {; } \\
& f\left(v_{i, 4}\right)=\frac{k i}{2}-k+4, \quad \text { if } i \text { is even, } 1 \leq i \leq n-1 \text {; } \\
& f\left(v_{i, j}\right)=\frac{k i}{2}-k+j+2, \quad \text { if } j \equiv 1(\bmod 4) \text { or } \\
& j \equiv 2(\bmod 4), 5 \leq j \leq k-2 \text {, } \\
& \text { and } i \text { is even, } 1 \leq i \leq n-1 \text {; } \\
& f\left(v_{i, j}\right)=\frac{k i}{2}-k+j+3, \quad \text { if } j \equiv 3(\bmod 4), 3 \leq j \leq k-2 \text {, } \\
& \text { and } i \text { is even, } 1 \leq i \leq n-1 \text {; } \\
& f\left(v_{i, j}\right)=\frac{k i}{2}-k+j+1, \quad \text { if } j \equiv 0(\bmod 4), 8 \leq j \leq k-2 \text {, } \\
& \text { and } i \text { is even, } 1 \leq i \leq n-1 \text {. }
\end{aligned}
$$

Then $e_{f^{\prime}}(0)=\left\lfloor\frac{(n-2) k+n}{4}\right\rfloor$ and $e_{f^{\prime}}(1)=\left\lceil\frac{(n-2) k+n}{4}\right\rceil$. 
Thus, $\left|e_{f^{\prime}}(0)-e_{f^{\prime}}(1)\right| \leq 1$.

Hence $A S_{n}^{3}\left(C_{k}\right)$ is $S D$-prime cordial, for $k \equiv 3(\bmod 4), k \geq 7$.

Theorem 2.13. The graph $A S_{n}^{1}\left(C_{k}\right)$ is $S D$-prime cordial, for $k \equiv 0(\bmod 4), k \geq 8$.

Proof 2.13. Define $f: V\left(A S_{n}^{1}\left(C_{k}\right)\right) \rightarrow\left\{1,2, \ldots, \frac{n k}{2}\right\}$ as follows:

$f\left(u_{i}\right)= \begin{cases}\frac{k i}{2}-\frac{k}{2}+1, & \text { if } i \text { is odd, } 1 \leq i \leq n ; \\ \frac{k i}{2}-1, & \text { if } i \text { is even, } 1 \leq i \leq n\end{cases}$

$f\left(v_{i, 1}\right)= \begin{cases}\frac{k i}{2}-\frac{k}{2}+5, & \text { if } i \equiv 1(\bmod 4), 1 \leq i \leq n-1 ; \\ \frac{k i}{2}-\frac{k}{2}+4, & \text { if } i \equiv 3(\bmod 4), 1 \leq i \leq n-1 ;\end{cases}$

$f\left(v_{i, 2}\right)= \begin{cases}\frac{k i}{2}-\frac{k}{2}+4, & \text { if } i \equiv 1(\bmod 4), 1 \leq i \leq n-1 ; \\ \frac{k i}{2}-\frac{k}{2}+3, & \text { if } i \equiv 3(\bmod 4), 1 \leq i \leq n-1 ;\end{cases}$

$f\left(v_{i, 3}\right)=\left\{\begin{array}{l}\frac{k i}{2}-\frac{k}{2}+3, \quad \text { if } i \equiv 1(\bmod 4), 1 \leq i \leq n-1 ; \\ \frac{h i}{2}-\frac{k}{2}+6,\end{array}\right.$

$f\left(v_{i, 3}\right)=\left\{\begin{array}{l}\frac{k i}{2}-\frac{k}{2}+6, \quad \text { if } i \equiv 3(\bmod 4), 1 \leq i \leq n-1 ;\end{array}\right.$

$f\left(v_{i, 4}\right)= \begin{cases}\frac{k i}{2}-\frac{k}{2}+2, & \text { if } i \equiv 1(\bmod 4), 1 \leq i \leq n-1 ; \\ \frac{k i}{2}-\frac{k}{2}+5, & \text { if } i \equiv 3(\bmod 4), 1 \leq i \leq n-1 ;\end{cases}$

$f\left(v_{i, 5}\right)= \begin{cases}\frac{k i}{2}-\frac{k}{2}+6, & \text { if } i \equiv 1(\bmod 4), 1 \leq i \leq n-1 ; \\ \frac{k i}{2}-\frac{k}{2}+2, & \text { if } i \equiv 3(\bmod 4), 1 \leq i \leq n-1\end{cases}$

$f\left(v_{i, j}\right)=\frac{k i}{2}-\frac{k}{2}+j+2, \quad$ if $j \equiv 2(\bmod 4), 6 \leq j \leq k-2$,

and $i$ is odd, $1 \leq i \leq n-1$;

$f\left(v_{i, j}\right)=\frac{k i}{2}-\frac{k}{2}+j, \quad$ if $j \equiv 3(\bmod 4), 7 \leq j \leq k-2$,

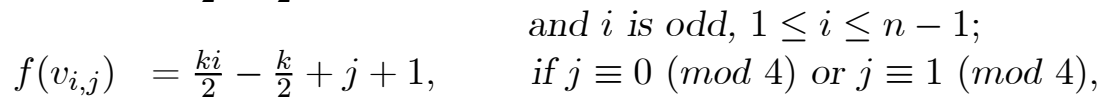

$8 \leq j \leq k-2$, and $i$ is odd, $1 \leq i \leq n-1$.

Then $e_{f^{\prime}}(0)=\left\lfloor\frac{n(k+1)-2}{4}\right\rfloor$ and $e_{f^{\prime}}(1)=\left\lceil\frac{n(k+1)-2}{4}\right\rceil$.

Thus, $\left|e_{f^{\prime}}(0)-e_{f^{\prime}}(1)\right| \leq 1$.

Hence $A S_{n}^{1}\left(C_{k}\right)$ is $S D$-prime cordial, for $k \equiv 0(\bmod 4), k \geq 8$.

Theorem 2.14. The graph $A S_{n}^{2}\left(C_{k}\right)$ is $S D$-prime cordial, for $k \equiv 0(\bmod 4), k \geq 8$. 
Proof 2.14. Define $f: V\left(A S_{n}^{2}\left(C_{k}\right)\right) \rightarrow\left\{1,2, \ldots, \frac{(n-1) k+2}{2}\right\}$ as per the previous theorem. Then $e_{f^{\prime}}(0)=\left\lceil\frac{(n-1)(k+1)}{4}\right\rceil$ and $e_{f^{\prime}}(1)=\left\lfloor\frac{(n-1)(k+1)}{4}\right\rfloor$.

Thus, $\left|e_{f^{\prime}}(0)-e_{f^{\prime}}(1)\right| \leq 1$.

Hence $A S_{n}^{2}\left(C_{k}\right)$ is $S D$-prime cordial, for $k \equiv 0(\bmod 4), k \geq 8$.

Theorem 2.15. The graph $A S_{n}^{3}\left(C_{k}\right)$ is $S D$-prime cordial, for $k \equiv 0(\bmod 4), k \geq 8$.

Proof 2.15. Define $f: V\left(A S_{n}^{3}\left(C_{k}\right)\right) \rightarrow\left\{1,2, \ldots, \frac{(n-1) k+4}{2}\right\}$ as follows:

$f\left(u_{i}\right)= \begin{cases}1, & \text { if } i=1 ; \\ \frac{k i}{2}-\frac{k}{2}, & \text { if } i \text { is odd, } 3 \leq i \leq n ; \\ \frac{k i}{2}-k+2, & \text { if } i \text { is even, } 1 \leq i \leq n\end{cases}$

$f\left(v_{i, 1}\right)= \begin{cases}\frac{k i}{2}-k+6, & \text { if } i \equiv 0(\bmod 4), 1 \leq i \leq n-1 \text { or } i=2 ; \\ \frac{k i}{2}-k+5, & \text { if } i \equiv 2(\bmod 4), 6 \leq i \leq n-1 ;\end{cases}$

$f\left(v_{i, 2}\right)= \begin{cases}\frac{k i}{2}-k+5, & \text { if } i \equiv 0(\bmod 4), 1 \leq i \leq n-1 \text { or } i=2 ; \\ \frac{k i}{2}-k+4, & \text { if } i \equiv 2(\bmod 4), 6 \leq i \leq n-1 ;\end{cases}$

$f\left(v_{i, 3}\right)= \begin{cases}\frac{k i}{2}-k+4, & \text { if } i \equiv 0(\bmod 4), 1 \leq i \leq n-1 \text { or } i=2 ; \\ \frac{k i}{2}-k+7, & \text { if } i \equiv 2(\bmod 4), 6 \leq i \leq n-1 ;\end{cases}$

$f\left(v_{i, 4}\right)= \begin{cases}\frac{k i}{2}-k+3, & \text { if } i \equiv 0(\bmod 4), 1 \leq i \leq n-1 \text { or } i=2 ; \\ \frac{k i}{2}-k+6, & \text { if } i \equiv 2(\bmod 4), 6 \leq i \leq n-1 ;\end{cases}$

$f\left(v_{i, 5}\right)= \begin{cases}\frac{k i}{2}-k+7, & \text { if } i \equiv 0(\bmod 4), 1 \leq i \leq n-1 \text { or } i=2 ; \\ \frac{k i}{2}-k+3, & \text { if } i \equiv 2(\bmod 4), 6 \leq i \leq n-1 ;\end{cases}$

$f\left(v_{i, j}\right)=\frac{k i}{2}-k+j+3, \quad$ if $j \equiv 2(\bmod 4), 6 \leq j \leq k-2$,

and $i$ is even, $1 \leq i \leq n-1$;

$f\left(v_{i, j}\right)=\frac{k i}{2}-k+j+1, \quad$ if $j \equiv 3(\bmod 4), 7 \leq j \leq k-2$,

and $i$ is even, $1 \leq i \leq n-1$;

$f\left(v_{i, j}\right)=\frac{k i}{2}-k+j+2, \quad$ if $j \equiv 0(\bmod 4)$ or $j \equiv 1(\bmod 4)$,

$8 \leq j \leq k-2$, and $i$ is even, $1 \leq i \leq n-1$. 
Then $e_{f^{\prime}}(0)=\left\lceil\frac{(n-2) k+n}{4}\right\rceil$ and $e_{f^{\prime}}(1)=\left\lfloor\frac{(n-2) k+n}{4}\right\rfloor$, for $n \geq 4$.

Thus, $\left|e_{f^{\prime}}(0)-e_{f^{\prime}}(1)\right| \leq 1$.

Hence $A S_{n}^{3}\left(C_{k}\right)$ is $S D$-prime cordial, for $k \equiv 0(\bmod 4), k \geq 8$.

\section{Illustration-3:}

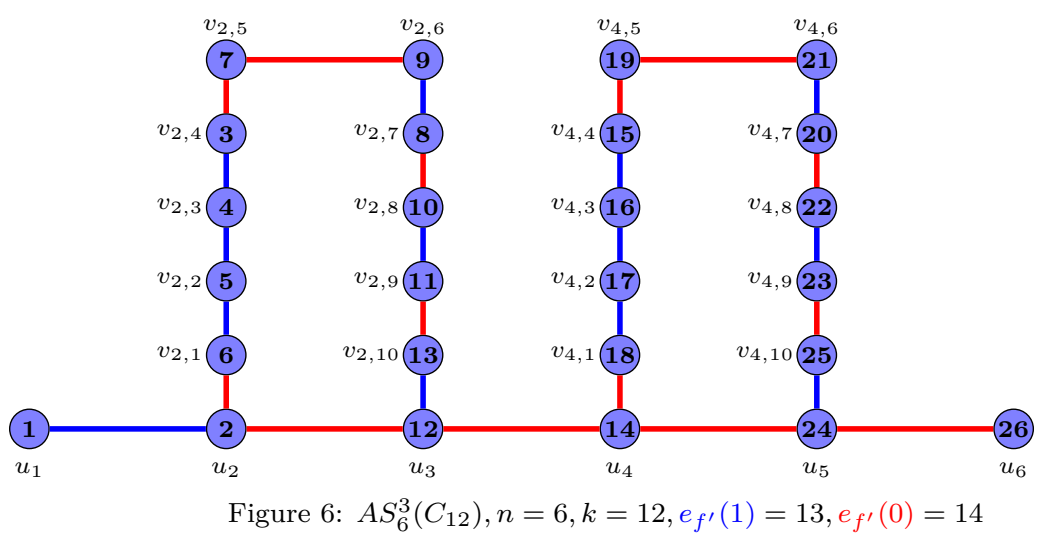

Theorem 2.16. The graphs $A S_{n}^{1}\left(C_{k}\right), A S_{n}^{2}\left(C_{k}\right)$ and $A S_{n}^{3}\left(C_{k}\right)$ are $S D$-prime cordial, for all integers $k \geq 3, n \geq 2$.

Proof 2.16. The proof follows from theorems 2.1 to 2.15 .

\section{Conclusions}

We have proved that each of the types of alternate k-polygonal Snake $A S_{n}\left(C_{k}\right)$ is SD-prime cordial, for all integers $k \geq 3, n \geq 2$. Further investigation can be done for other graph families. For instance, one may consider cyclic ladder graph. 


\section{References}

[1] J. A. Bondy and U. S. R. Murty, Graph theory with applications. London: Macmillan, 1976. [On line]. Available: https:/ / bit.ly/ 3xhcnfP

[2] J. A. Gallian, "A dynamic survey of graph labeling", The electronic journal of combinatorics, DS6, 2019, doi: 10.37236/ 27

[3] G.-C. Lau, H.-H. Chu, N. Suhadak, F.-Y. Foo, and H.-K. Ng, "On SD-prime cordial graphs", International journal of pure and apllied mathematics, vol. 106, no. 4, pp. 1017-1028, 2016, doi: 10.12732/ ijpam.v106i4.4

[4] A. Lourdusamy and F. Patrick, "Some results on SD-prime cordial labeling", Proyecciones (Antofagasta), vol. 36, no. 4, pp. 601-614, 2017, doi: 10.4067/ s0716-09172017000400601

[5] A. Lourdusamy, S. J. Wency, and F. Patrick, "On SD-prime cordial labeling", International journal of pure and applied mathematics, vol. 117, special no. 11, pp. 221-228, 2017. [On line]. Available: https:/ / bit.ly/ 3vbzHKf

[6] V. Ramachandran and C. Sekar. "One modulo $N$ gracefulness of $n$-polygonal snakes, $C_{n}^{(t)}$ and $P_{a, b}$ ", International journal of engineering research and technology (Ahmedabad), vol. 2, no. 10, pp. 3514-3529, 2013. [On line]. Available: https:/ / bit.ly/ 32H4t1w

[7] U. M. Prajapati and K. P. Shah, "Odd prime labeling of various snake graphs", International journal of scientific research and reviews, vol. 8, no. 2, pp. 2876-2885, 2019. [On line]. Available: https:/ / bit.ly/ 2RW69lA

[8] U. M. Prajapati and A. V. Vantiya, "SD-prime cordial labeling of some snake graphs", Journal of applied science and computations, vol. 6, no. 4, pp. 1857-1868, 2019. [On line]. Available: https:/ / bit.ly/ 3ayUDTE

[9] U. M. Prajapati and A. V. Vantiya, "SD-prime cordial labeling of subdivision of snake graphs", International journal of scientific research and reviews, vol. 8, no. 2, pp. 2414-2423, 2019. [On line]. Available: https:// bit.ly/ 3enIulu 